\title{
INFLUÊNCIA DE GRADIENTES GEOGRÁFICOS NA ESTIMATIVA DO ESTOQUE DE CARBONO EM FLORESTA ESTACIONAL SEMIDECIDUAL
}

\author{
INFLUENCE OF GEOGRAPHIC GRADIENTS IN THE ESTIMATE OF CARBON STOCK A \\ SEASONAL SEMIDECIDAL FOREST
}

\author{
Hortênsia Vilas Bôas ${ }^{1}$, Evandro Nunes Miranda², Laís Almeida Araújo ${ }^{3}$, Mônica Canaan \\ Carvalho ${ }^{4}$, Kallil José Viana da Páscoa ${ }^{5}$, Lucas Rezende Gomide 6 \\ 1, 2, 3, 4, 5, 6 Universidade Federal de Lavras, Lavras, Minas Gerais, Brasil - hortensiavillas123@gmail.com, \\ evandromiranda.florestal@gmail.com; la_sal@hotmail.com; monicacanaan@gmail.com, \\ kalillpascoa@gmail.com \& lucasgomide@ufla.br
}

\begin{abstract}
RESUMO
Diante da importância das formações florestais na fixação de $\mathrm{CO}_{2}$ da atmosfera faz-se necessário o conhecimento da variação do estoque de carbono nos diferentes ecossistemas. Assim, objetivou-se caracterizar a composição florística, e a estrutura do componente arbóreo, avaliar a relação entre as variáveis dendrométricas do povoamento e os gradientes para o estoque do carbono presente no compartimento arbóreo-aéreo, além de desenvolver um modelo linear múltiplo de regressão para explicar os estoques de carbono em um fragmento de Floresta Estacional Semidecidual. No local foram alocadas 57 parcelas medindo as variáveis CAP (circunferência à altura do peito) e altura total dos indivíduos acima de $15 \mathrm{~cm}$. Os gradientes avaliados foram: longitude, latitude, altitude e distância do rio para cada parcela. $O$ estoque de carbono foi estimado pela equação própria da região. Além disso, no entendimento das relações entre variáveis foi aplicada uma análise de componente principal (PCA) para identificar as variáveis de maior contribuição na explicativa do estoque de carbono. Os resultados mostram um fragmento com alta densidade de indivíduos ( $1.580 \mathrm{~N} / \mathrm{ha}$ ) e 160 espécies. As cinco espécies com maior valor de importância foram: Piptadenia gonoacantha, Senegalia polyphylla, Machaerium stipitatum, Actinostemon verticillatus e Trichilia casaretti. A PCA permitiu observar que os gradientes estão altamente correlacionados com a distribuição do estoque de carbono do compartimento aéreo da vegetação. Na composição do modelo para estoque de carbono as variáveis selecionadas foram: área basal, número de indivíduos, longitude e altitude. A longitude e altitude contribuíram significativamente na melhoria do modelo proposto.
\end{abstract}

PALAVRAS-CHAVE: Análise de componentes principais, Fitossociologia, Modelagem.

\section{ABSTRACT}

Given the importance of forest formations in fixation of the $\mathrm{CO}_{2}$ from the atmosphere, it is necessary the knowledge of carbon variation in different ecosystems. Thus, the objective of this study was to characterize a floristic composition and a structure of the tree component, to evaluate a relationship between dendrometric variables of the stands and the gradients for the carbon storage present in the aboveground tree compartment, in addition to developing a linear multiple regression model to explain the carbon storages in a fragment of Seasonal Semideciduous Forest. At the site, 57 plots were allocated measuring the variables CAP (circumference at breast height) and total height of $15 \mathrm{~cm}$ above. The gradients evaluated were: longitude, latitude, altitude, and distance of the river for each plot. The carbon storage was estimated by the region's own equation. In addition, the differences between the variables were applied as a Principal component analysis (PCA) to identify the variables of greatest contribution in explaining the carbon storage. The results show a fragment with a high density of individuals $(1,580 \mathrm{~N} / \mathrm{ha})$ and 160 species. The five species with the highest importance value were: Piptadenia gonoacantha, Senegalia polyphylla, Machaerium stipitatum, Actinostemon verticillatus, and Trichilia casaretti. PCA allowed observing that the gradients are highly correlated with the distribution of carbon storage from the aboveground compartment of the vegetation. On the composition of the carbon storage model the variables selected were: basal area, number of individuals, longitude, and altitude. Longitude and altitude significantly contributed significantly to the improvement of the proposed model.

KEYWORDS: Principal component analysis, Phytosociology, Modeling. 


\section{INTRODUÇÃO}

A Floresta Estacional Semidecidual é uma fisionomia pertencente ao bioma Mata Atlântica, que por sua vez engloba diferentes ecossistemas, estando associada desde a áreas de mangues, enseadas, fozes de grandes rios, baías, baixadas arenosas, florestas mistas de araucárias a campos de altitude e rupestre. Esses ecossistemas apresentam variações nas formações vegetais sem perder a homogeneidade florística, sendo influenciada por aspectos edafoclimáticos (BARBOSA \& THOMAS, 2002).

Esse bioma brasileiro apresenta grande diversidade de espécies, abrangendo áreas consideradas como as de maior biodiversidade do planeta. No entanto, a Mata Atlântica vem sofrendo uma histórica redução em sua cobertura vegetal devido às atividades antrópicas, principalmente associadas à substituição das áreas cobertas por vegetação natural por áreas agrícolas (VICHARNAKORN et al., 2014), sendo essa uma das principais causas da intensificação de liberação dos gases de efeito estufa, como o dióxido de carbono (MORAIS et al., 2017; DELGADO et al., 2018).

Dessa maneira, as formações florestais desenvolvem um papel importante na fixação de $\mathrm{CO}_{2}$ da atmosfera, auxiliando no equilíbrio do ciclo de carbono por meio da sua fixação na biomassa das plantas durante seu crescimento (RATUCHNE et al., 2015).

Assim, é evidente que as plantas contribuem de forma direta para redução na emissão de gases e consolida a importância da quantificação do estoque de carbono fixado nas florestas (SILVA et al., 2018). Além disso, ter conhecimento do potencial de se estocar carbono pelas florestas pode auxiliar em programas de comercialização e créditos de carbono, bem como incentivos ao reflorestamento de áreas degradadas (SANTOS et al., 2016).

Diante da importância das formações florestais na fixação de $\mathrm{CO}_{2}$ da atmosfera faz-se necessário o conhecimento da variação do estoque de carbono nos diferentes ecossistemas. Assim, os objetivos desse trabalho foram: a) caracterizar a composição florística e a estrutura do componente arbóreo; b) avaliar a relação entre as variáveis dendrométricas do povoamento e os gradientes ambientais com o estoque do carbono presente no compartimento aéreo e; c) desenvolver um modelo de regressão linear múltipla para explicar os estoques de carbono no local de estudo.

\section{MATERIAL E MÉTODOS}

\section{Caracterização da área de estudo}

O fragmento estudado está localizado na Fazenda do Faria, no município de Nepomuceno, na região Sul de Minas Gerais e às margens do Rio Grande (2106'36.66"S e $\left.45^{\circ} 10^{\prime} 12^{\prime \prime} \mathrm{W}\right)$, conforme apresentado na Figura 1 . A área total do fragmento é de 37,77 hectares, classificados como Floresta Estacional Semidecidual Sub Montana (CARVALHO \& SCOLFORO, 2008). A altitude da área varia de 780 a 840 metros, e de acordo com a classificação de Köppen o clima da área é o CWB, com verões brandos e suaves, e estiagem no inverno (ALVARES et al., 2013).

\section{Inventário florestal}

As unidades amostrais foram sistematizadas na área, onde primeiro foram lançados transectos distantes 100 metros entre si e ao longo do fragmento, totalizando 6 transectos. Individualmente, cada transecto recebeu conglomerados formados por três elementos (parcelas) de $10 \mathrm{~m} \times 25 \mathrm{~m}\left(250 \mathrm{~m}^{2}\right)$, distantes $25 \mathrm{~m}$ entre si, sendo os conglomerados distanciados $50 \mathrm{~m}$ um do outro, totalizando 24 conglomerados (Figura 1).

Em cada parcela foram inventariados todos os indivíduos que apresentaram circunferência medida a 1,3 $\mathrm{m}$ de altura do solo (CAP) igual ou superior a $15,7 \mathrm{~cm}$ (diâmetro igual ou superior a $5 \mathrm{~cm}$ ). Foram mensurados o CAPs e as alturas totais $(\mathrm{H})$ de cada indivíduo amostrado por meio da fita métrica e da vara telescópica, respectivamente. As espécies arbóreas, cujo nome científico, não foram identificadas no momento do inventário, tiveram material botânico coletado para posterior identificação por especialistas da área.

\section{Fitossociologia, análise dos gradientes e variáveis do povoamento}

A avaliação dos parâmetros fitossociológicos foi realizada com base nos valores tanto absolutos quanto relativos de densidade (DA e DR), dominância (DoA e DoR), frequência (FA e FR) e o valor de importância (VI). Em relação à diversidade florística, foram utilizados os índices de Shannon $\left(\mathrm{H}^{\prime}\right)$ e equabilidade de Pielou ( $\mathrm{J}^{\prime}$ ).

As variáveis do povoamento como área basal (G), número de indivíduos ( $\mathrm{N}$ ) e riqueza de espécies (Esp) foram geradas a partir dos dados dendrométricos mensurados durante o inventário. $O$ estoque de carbono individual foi calculado pela Equação (1) específica para a região, com erro padrão dos resíduos Syx (Mg.ha-1) de 0,15538, Syx (\%) de 36,4 e R $^{2}$ aj de $97,25 \%$ (SCOLFORO et. al, 2008). 


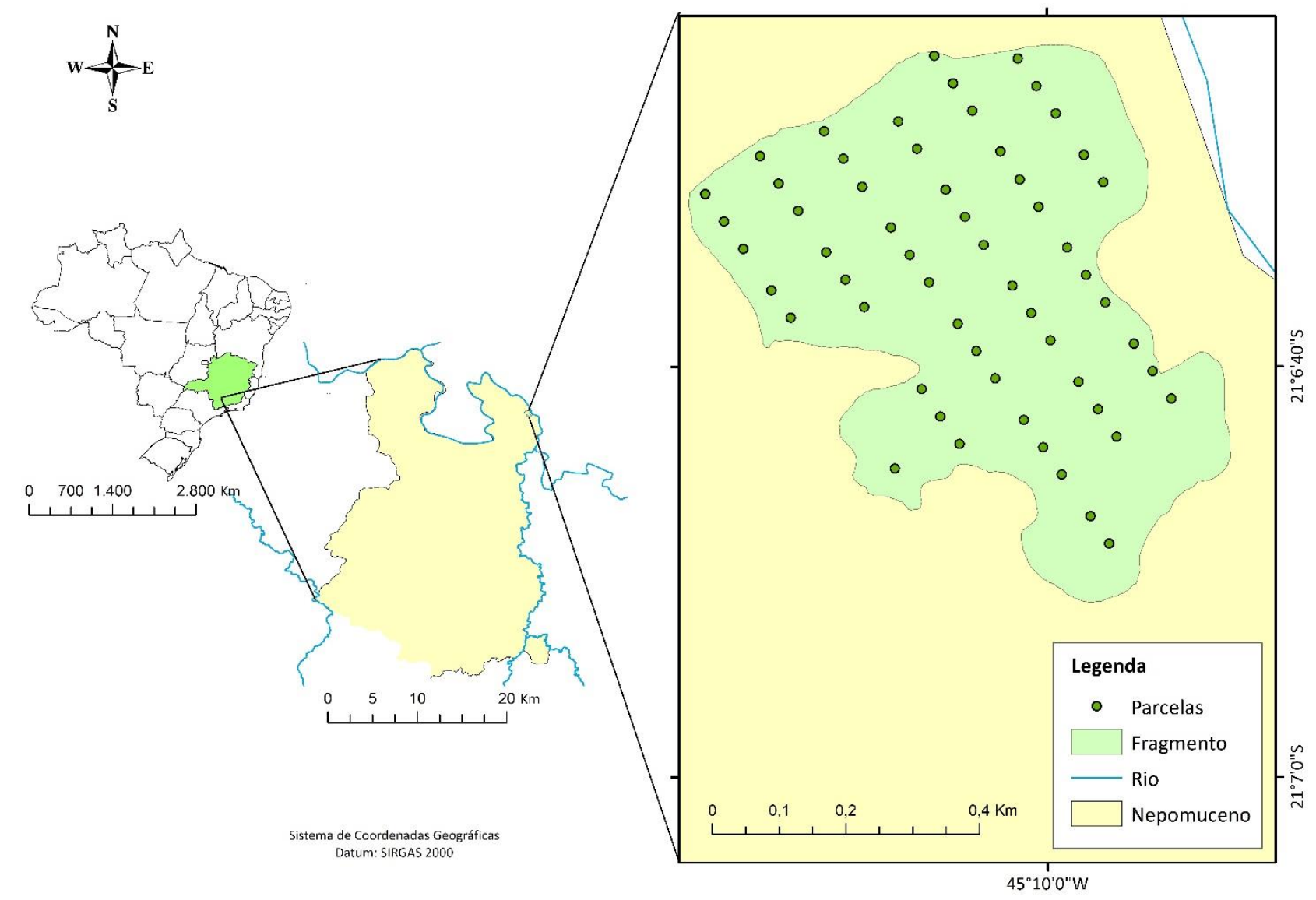

Figura 1. Mapa da área de estudo com a disposição das parcelas do inventário florestal.

$$
\operatorname{Ln}(C)=-12,303439+2,6584231 * \ln (D A P)+0,571171 * \ln (H)
$$

Em que: $\mathrm{C}=$ quantidade de carbono; $\mathrm{DAP}=$ diâmetro medido a $1,3 \mathrm{~m}$ de altura $\geq 5 \mathrm{~cm}$; e $\mathrm{H}=$ altura total de cada indivíduo amostrado.

Os gradientes coletados para compor a análise foram longitude, latitude, altitude e distância ortogonal do eixo do rio. Assim, após serem lançadas as parcelas na área, foram coletadas, com o uso do GPS, a longitude (X) e a latitude (Y) no Sistema de Coordenadas Geográfica e Datum SIRGAS 2000, e a altitude na área central de cada parcela. Posteriormente, a distância entre o rio e cada parcela foram medidas considerando a distância euclidiana entre a parcela e a rede hidrográfica, por sua medida ortogonal.

\section{Modelagem do estoque de carbono da parte aérea (ECPA) e dinâmica dos gradientes com o carbono}

Uma análise dos componentes principais (PCA) foi realizada para verificar quais principais variáveis do povoamento e dos gradientes que são mais explicativas no processo da modelagem do estoque de carbono. O pacote utilizado foi o factoextra (KASSAMBARA \& MUNDT, 2017) do software R (Version 3.5.2 - (C) 2018 RStudio, Inc.). A técnica PCA evita a perda de informação das variáveis e possíveis problemas com multicolinearidade (FELFILI et al., 2007; TERRA et al., 2015). Desse modo, as variáveis mais explicativas da primeira componente principal (PC1) e da segunda (PC2) foram utilizadas como variáveis de entrada no modelo de regressão linear múltipla. O referido modelo busca explicar o estoque de carbono da parte aérea (ECPA) existente na área, ou seja, um modelo em nível de povoamento com adição livre de variáveis dos gradientes ambientais observados. Esses gradientes ambientais foram selecionados de acordo com os resultados obtidos na PCA.

\section{Análise estatística}

O modelo gerado teve sua significância testada por teste $F(\alpha=0,05)$, bem como a significância de seus parâmetros pelo teste $\mathrm{t}(\alpha=0,05)$. De modo a avaliar o modelo desenvolvido adotou-se estatísticas clássicas de precisão, tais como: erro padrão residual (Syx), erro padrão residual em porcentagem (Syx \%), gráfico de resíduo padronizado, gráfico de correlação, coeficiente de determinação ajustado ( $\left.R^{2} a j\right)$ e o fator de inflação da variância (VIF). Esse último, adotou-se um critério de VIF > 
5, sendo excluído variáveis/parâmetros maiores que o máximo admitido. $\mathrm{O}$ modelo foi ajustado no software $\mathrm{R}$ (Version 3.5.2 - (C) 2018 RStudio, Inc.), considerando o método dos mínimos quadrados ordinais.

\section{RESULTADOS E DISCUSSÃO}

\section{Fitossociologia}

Através da análise fitossociológica da área de estudo, encontrou-se as cinco espécies com maior densidade relativa: Actinostemon verticillatus (Klotzsch) Baill. (8,86\%), Trichilia casaretti C.DC. (5,76\%), Senegalia polyphylla (DC.) Britton \& Rose $(5,76 \%)$, Machaerium stipitatum Vogel $(5,57)$ e Piptadenia gonoacantha (Mart.) MACBR (5,25\%). Entre as cinco espécies de maior densidade, quatro também estão entre as mais frequentes, são elas: Machaerium stipitatum (4,39\%), Senegalia polyphylla (4,13\%), Piptadenia gonoacantha (4,00\%) e Trichilia casaretti (3,10\%). Os cincos maiores valores de dominância relativa, ou seja, os maiores valores em área basal (G) foram encontrados para as seguintes espécies: Piptadenia gonoacantha (14,75\%), Senegalia polyphylla (7,89\%), Machaerium stipitatum (4,37\%), Platycyamus regnellii $(4,10 \%)$ e Cariniana legalis (3,88\%). Entre essas cinco de maior dominância, três delas (Piptadenia gonoacantha, Senegalia polyphylla e Machaerium stipitatum) estão entre as espécies de maior densidade no fragmento.

Em florestas tropicais ocorre um grande número de espécies com valores baixos de densidade (VIANA et al., 2016). Este fato foi observado na área estudada, uma vez que, das 160 espécies amostradas, 105 espécies (65,63\% do total) apresentaram densidade igual ou inferior a cinco indivíduos e 49 espécies (30,62\% do total) apresentaram densidade igual a um indivíduo. De acordo com Lyons et al. (2005), mesmo pouco abundantes, as espécies com baixa densidade são importantes para garantir equilíbrio ao funcionamento do ecossistema, desempenhando funções como aumentar resistência da comunidade contra plantas invasoras e retenção de nutrientes.

As cinco espécies arbóreas que apresentaram maior valor de importância (VI) (Figura 2) estão entre as cinco espécies de maior densidade, são elas em ordem decrescente: Piptadenia gonoacantha, Senegalia polyphylla, Machaerium stipitatum, Actinostemon verticillatus e Trichilia casaretti. Dessas cinco espécies de maior VI, as espécies Piptadenia gonoacantha, Senegalia polyphylla e Machaerium stipitatum pertencem à família Fabaceae, sendo espécies arbóreas nativas, não endêmicas e encontradas na Mata Atlântica. Embora essas espécies ocorram em vários tipos de vegetação, elas são típicas da Floresta Estacional Semidecidual, que ocorre no Sudeste e outras regiões do país. De acordo com Silva \& Leitão Filho (1982), a família Fabaceae geralmente é a família que domina o estrato arbóreo das florestas tropicais do Brasil.

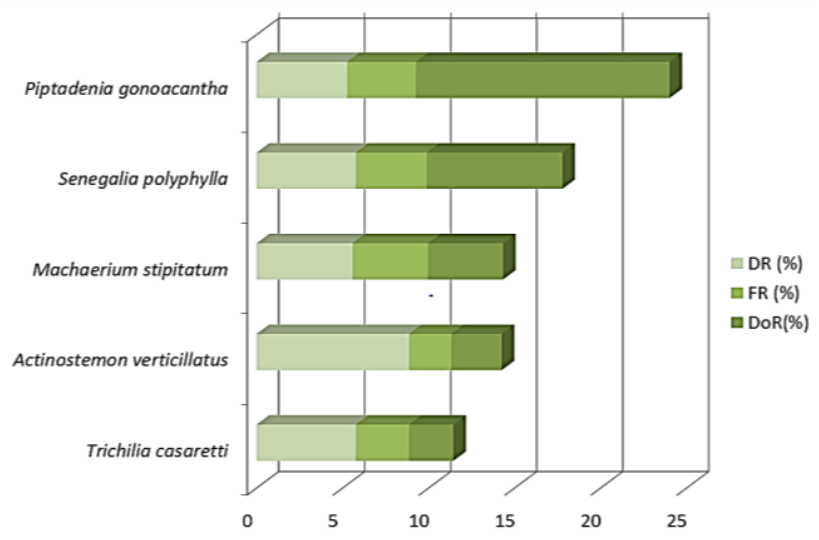

Figura 2. Relação das cinco espécies de maior valor de importância (VI (\%)) encontradas no fragmento florestal na Fazendo do Faria, Nepomuceno-MG.

As espécies Actinostemon verticillatus e Trichilia casaretti pertencem respectivamente às famílias Euphorbiaceae e Meliaceae, são espécies nativas e encontradas no bioma Mata Atlântica. A espécie Actinostemon verticillatus é característica de florestas ombrófilas, mas devido ao fragmento analisado se localizar em uma área de transição entre as fisionomias sua ocorrência pôde ser observada. Já a espécie Trichilia casaretti ocorre em diferentes tipos de vegetação, sendo uma delas a floresta estacional; ambas ocorrem no Sudeste e outras regiões (Reflora - Herbário Virtual).

Pode-se observar que a espécie Piptadenia gonoacantha apresentou o maior VI (24\%), devido principalmente a dominância relativa DoR (14,75\%), sendo a espécie que apresentou o maior valor de DoR e o quinto maior valor de DR (5,25\%). A espécie Trichilia casaretti apresentou o menor VI (11,43\%) e consequentemente o menor valor de DoR (2,58\%). Entre as cinco espécies de maior VI, foi possível observar que o VI e o valor de DoR estão diretamente relacionados. Dentre as cinco espécies de maior VI, a espécie que apresentou o menor VI (Trichilia casaretti), possui o mesmo valor de DR da espécie que apresentou o segundo maior VI (Senegalia polyphylla), fato que demostra que a DoR foi o parâmetro que ocasionou está diferença no VI entre as duas espécies. Já a espécie Actinostemon verticillatus apresentou o maior valor de DR $(8,86 \%)$, porém sua DoR (2,93\%) não está entre os cinco maiores valores.

Archanjo (2008) estudando um fragmento de mata 
atlântica relata que ao classificar as espécies de maior valor de importância, o parâmetro FR teve pouca influência nesta classificação, principalmente nas espécies de maior $\mathrm{VI}$, sendo os maiores VI devido, principalmente, aos elevados valores de $D R$ e assim não sendo tão preponderante em termos de dominância na comunidade florestal. Em relação à $F R$, este relato foi observado neste trabalho, uma vez que o valor de FR das 5 espécies de maior VI não apresentou variações significativas entre estas espécies, já em relação a DR e a DoR ocorreu o inverso, uma vez que a DoR foi o parâmetro que mais influenciou nos resultados, mostrando assim que na área em estudo há um significativo grau de ocupação destas espécies na floresta.

O número de espécies raras atingiu $60 \%$ da lista total, apresentando valor de importância (VI) menor que 1\%. De acordo com Martins (1979), a ocorrência de muitas espécies com baixo VI é uma característica de florestas tropicais. Por outro lado, a diversidade, em floresta estacional quantificada pelo índice de Shannon chega a valores próximos de 3,98, o que representa uma alta diversidade (GIÁCOMO et al., 2013), assim pode-se considerar que o resultado encontrado para o índice de Shannon $(4,11)$ na área do fragmento é expressivo em relação a florestas estacionais.

$\mathrm{O}$ valor encontrado para a equabilidade de Pielou ( $\left.\mathrm{J}^{\prime}\right)$ foi de 0,81 , isto indica que considerando a diversidade máxima teórica se tem $81 \%$ dela representada nesta amostragem. Em Minas Gerais para Florestas Estacionais, o índice de diversidade $\left(H^{\prime}\right)$ varia entre 3,2 e 4,2 e já a equabilidade $\left(J^{\prime}\right)$ varia entre 0,73 e 0,88 (MEIRA NETO \& MARTINS, 2000). Dessa forma é possível observar que o índice de diversidade e equabilidade estão dentro da faixa de variação para o tipo de formação vegetal.

\section{Correlações entre os gradientes, as variáveis do povoamento e o carbono aéreo}

Ao estudar a relação entre estoque de carbono da parte aérea com variáveis do povoamento e gradientes locais, utilizando a análise dos componentes principais foi possível verificar qual é a contribuição de cada variável na predição de estoque de carbono. Na Figura 3 é apresentado as tendências dos gradientes no fragmento.
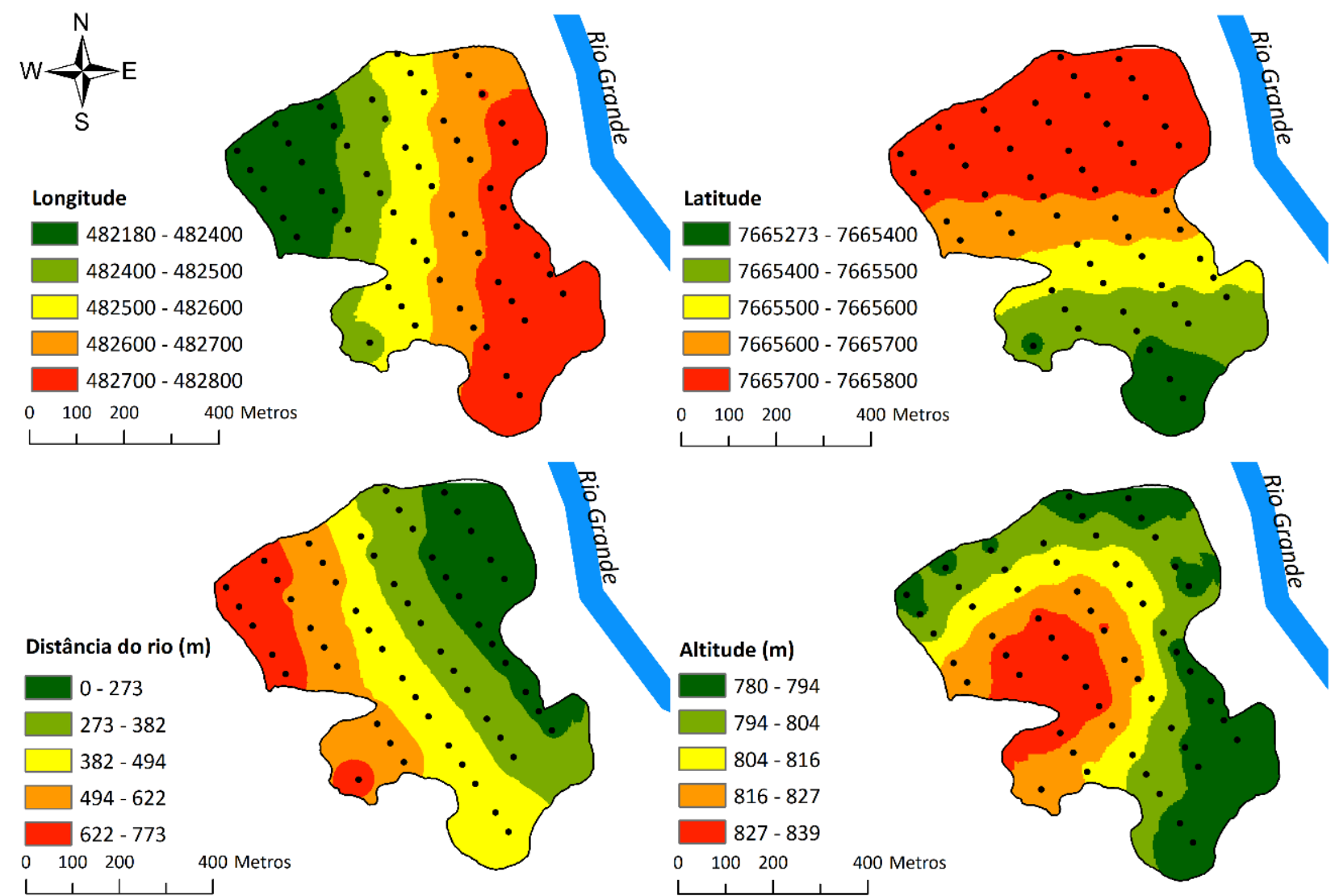

Figura 3. Mapa de distribuição espacial dos gradientes ambientais ao longo do fragmento florestal. 


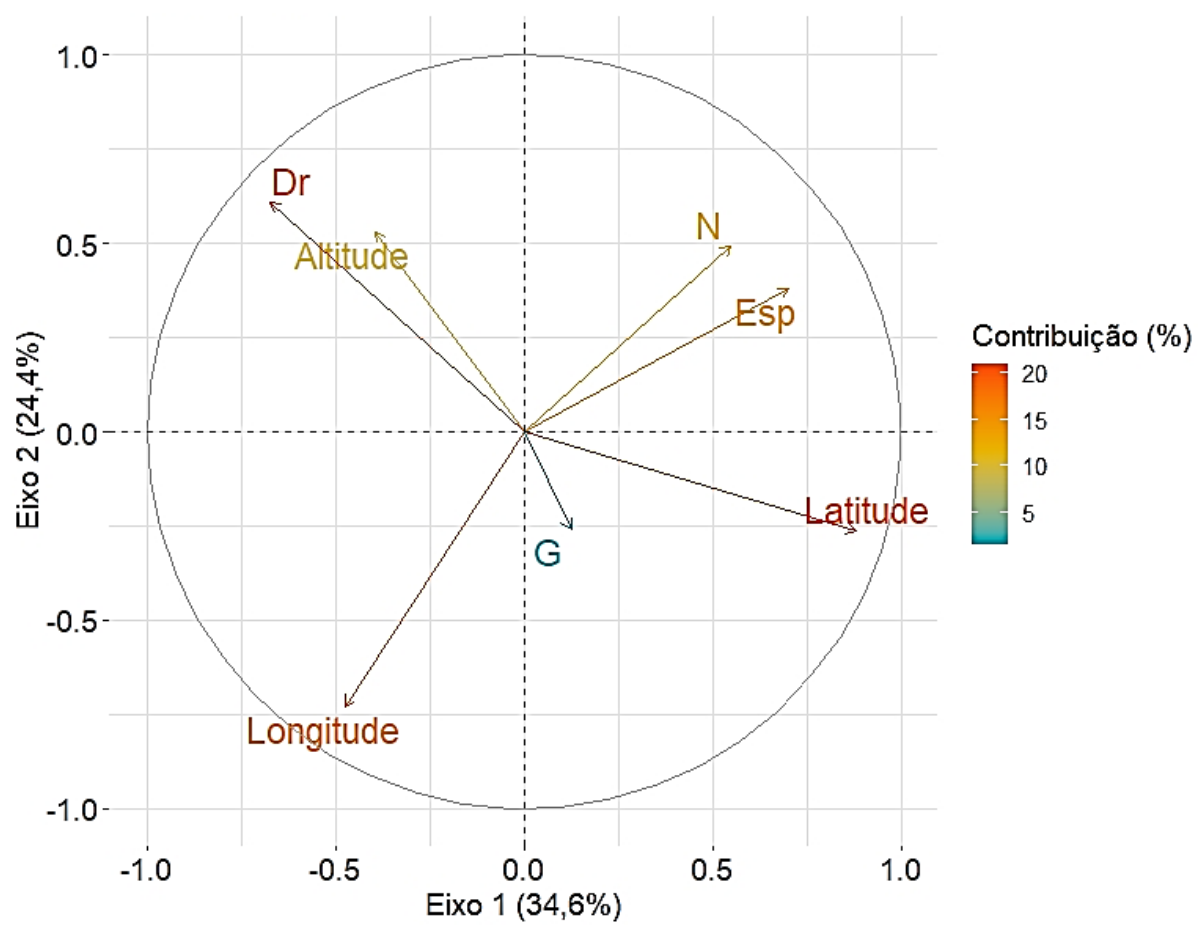

Figura 4. Gráfico de análise de componentes principais de variáveis relacionadas ao estoque de carbono da parte aérea em fragmento de Mata Atlântica no município de Nepomunceno - MG.

Em que: Área basal (G); densidade (N); riqueza de espécies (Esp); altitude (Alt); distância do rio (Dr); longitude; atitude; Eixo 1: porcentagem de explicação da componente principal 1 para a variabilidade dos dados $(24,4 \%)$; Eixo 2: porcentagem de explicação da componente principal 2 para a variabilidade dos dados $(24,4 \%)$.

Ao se analisar a primeira componente principal, verifica-se que as variáveis latitude e riqueza de espécies apresentaram os maiores autovetores positivos, $(56,71 \% \mathrm{e}$ $45,18 \%$ respectivamente), já os autovetores negativos mais significativos referem-se a distância do rio e longitude $(43,21 \%$ e $30,55 \%$ respectivamente). Higuchi et al. (2012) constataram que o componente arbóreo mostrou alta relação entre diversidade florística e estrutural com variáveis químicas do solo e topográficas. Também estudando a influência da diversidade florística, Poorter et al. (2015) observaram que áreas com alta diversidade tendem a ter uma alta quantidade de biomassa, indicando serem áreas com alto potencial de armazenamento de carbono.

Quanto à componente principal 2 (Eixo 2), as variáveis altitude e distância do rio obtiveram os maiores autovetores positivos $(46,68 \%$ e $40,54 \%$, respectivamente), e a longitude e latitude os maiores autovetores negativos $(55,76 \%$ e $\quad 20,12 \%$ respectivamente). Terra et al. (2015) ao estudarem a influência da altitude, fatores edafoclimáticos e topográficos no estoque de carbono e na dinâmica da vegetação em um fragmento de Mata Atlântica na Serra da Mantiqueira - MG, aplicando a técnica de PCA, encontraram maior correlação entre a altitude e os atributos da vegetação.

Diante dos autovetores obtidos pode-se afirmar que as duas primeiras componentes principais englobam características mais plausíveis para explicar a variabilidade do estoque de carbono no fragmento estudado. Sendo assim, a análise de componentes principais demonstrou um bom desempenho em correlacionar as variáveis do povoamento e gradientes com o estoque de carbono da parte aérea.

\section{Ajuste do modelo para estoque de carbono}

A partir das variáveis que demonstraram maior contribuição na análise de componentes principais (latitude, longitude, distância do rio, altitude e riqueza de espécies) e as variáveis do povoamento, foi analisada a significância dessas pelo teste $\mathrm{F}$ para a composição do modelo final (Equação 2):

$$
C_{i}=\beta_{0}+\beta_{1} N+\beta_{2} \text { Long }+\beta_{3} A l t+\beta_{4} G+\varepsilon_{i}
$$

Em que: $C$ = estoque de carbono da parte aérea na amostra i; $\mathrm{N}=$ número de indivíduos da espécie por ha; Long = longitude; Alt = altitude; $\mathrm{G}$ = área basal por hectare; $\beta_{i}=$ parâmetros a serem estimados; $e_{i}=$ erro aleatório da amostra i. 
Ao analisar as medidas de precisão referente ao modelo (Tabela 1), foi encontrado $R^{2}$ aj de $98,6 \%$ em relação a variação do estoque de carbono da parte aérea. Quanto ao Syx e Syx (\%) observou-se que o modelo

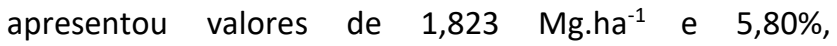
respectivamente. Uma análise de multicolinearidade entre as variáveis foi realizada e com base no VIF inferior a 5, as mesmas podem ser utilizadas, já que não apresentam multicolinearidade.

Parras-Alcántara et al. (2015), observaram que ocorre uma correlação negativa do teor de $\mathrm{CO}_{2}$ com a altitude, desse modo, à medida que o gradiente altitudinal aumenta ocorre uma diminuição do valor do estoque de carbono, o que pode ser atribuído a maior disponibilidade de nutrientes nas menores altitudes. No entanto, Terra et al. (2015) ao estudarem um fragmento de Mata Atlântica na Serra da Mantiqueira-MG, sobre Influência de variáveis topográficas e edafoclimáticas, constataram que o gradiente altitudinal pode influenciar positivamente no estoque de carbono. Assim como em Tsozué et al., (2019), que observaram relação direta entre o estoque de Carbono e o gradiente altitudinal. Do mesmo modo, o modelo gerado neste estudo também evidenciou correlação positiva da altitude na predição do estoque de carbono, ou seja, à medida que se aumenta a altitude, o estoque de

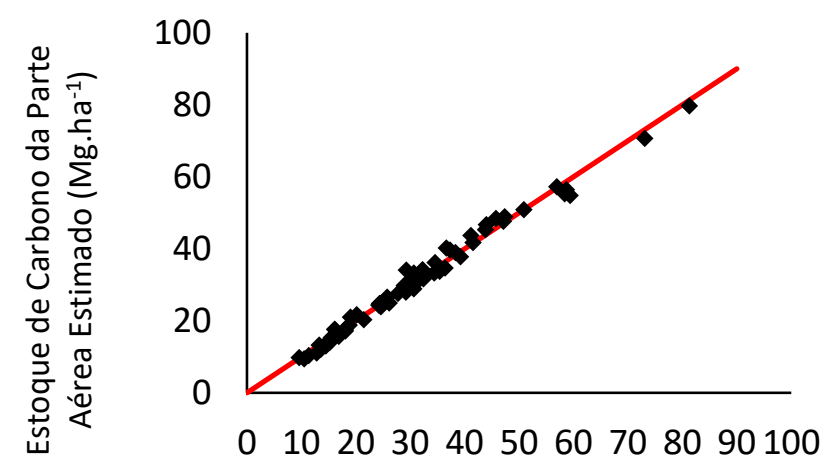

Estoque de Carbono da Parte Aérea Observado (Mg.ha-1) carbono da parte aérea aumenta conforme elevação do terreno.

Tabela 1. Análise descritiva do modelo e suas estatísticas na predição do estoque de carbono da parte aérea (ECPA).

\begin{tabular}{|c|c|c|c|}
\hline \multicolumn{3}{|c|}{ Análises } & Modelo \\
\hline \multirow{5}{*}{ Parâmetros } & & $\beta_{0}$ & 27460 \\
\hline & & $\beta_{1}$ & $-0,005276$ \\
\hline & & $\beta_{2}$ & $-0,003586$ \\
\hline & & $\beta_{3}$ & 0,03504 \\
\hline & & $\beta_{4}$ & 2,233 \\
\hline \multirow{3}{*}{ I } & \multicolumn{2}{|c|}{ Syx (Mg.ha-1) } & 1,823 \\
\hline & \multicolumn{2}{|c|}{ Syx (\%) } & 5,805 \\
\hline & \multicolumn{2}{|c|}{$\mathrm{R}^{2}{ }_{\mathrm{aj}}(\%)$} & 98,6 \\
\hline \multirow{4}{*}{ II } & \multirow{4}{*}{ VIF } & $N^{*}$ & 1,33 \\
\hline & & Longitude* & 1,35 \\
\hline & & Altitude* & 1,05 \\
\hline & & $\mathrm{G}^{*}$ & 1,21 \\
\hline
\end{tabular}

$\mathrm{I}=$ critérios de avaliação dos modelos; II = análise das variáveis dos modelos; $\beta i=$ parâmetros estimados; $\mathrm{Syx}=$ erro padrão residual; Syx\% = erro padrão residual em porcentagem; $\mathrm{R}^{2}{ }_{\mathrm{aj}}=$ coeficiente de determinação ajustado; VIF = fator de inflação da variância; $\mathrm{N}=$ densidade; $\mathrm{G}$ = área basal; $\mathrm{e}^{*}$ = significativo ao nível de probabilidade de $95 \%$

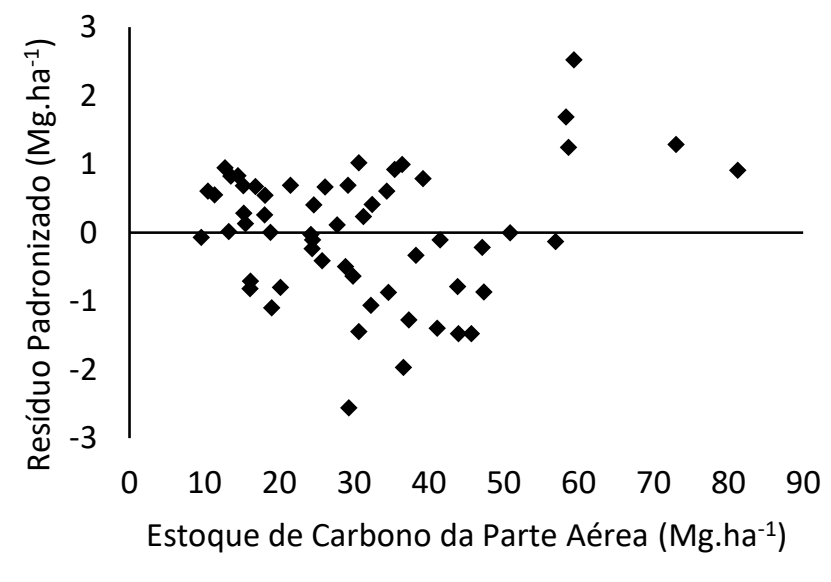

Figura 5. Gráficos de correlação e resíduo padronizado para o estoque de carbono da parte aérea.

Na Figura 5 são apresentados a distribuição do estoque de carbono da parte aérea estimada em relação ao estoque observado e o erro padronizado. O modelo ajustado mostrou resíduos com distribuição normal, homocedasticidade com o intervalo entre 3 e -3. Além disso, associado às estatísticas de precisão foi verificado ao longo da linha de tendência, a correlação entre os valores estimados e observados do fragmento. Devido a esses resultados avaliados obtêm-se estimativas mais precisas, o que proporciona menor invariabilidade nas estimativas. Além disso, a adição das variáveis geográficas ao modelo demonstrou-se significativa, destacando a influência da altitude e da longitude nas estimativas. Assim, foi confirmada a influência dos gradientes espaciais, os quais são capazes de carregar consigo efeitos que ditam a capacidade produtiva e os estoques de carbono.

Resultados de estoques de carbono em áreas de paisagens fragmentadas exigem a necessidade de se 
incorporar estimativas de carbono em escalas espaciais (longitude, latitude, altitude) mais refinadas, uma vez que apresentam alta variabilidade climática (ROMITELLI, 2014). Além disso, Wang et al. (2010) afirmam que para obtenção de estimativas de carbono mais precisas é necessário informações envolvendo gradientes ambientais e em diferentes escalas. Logo, percebe-se que ganhos nos modelos em nível de povoamento são identificados com a introdução dessas variáveis.

\section{CONCLUSÕES}

O fragmento analisado apresentou alta diversidade de espécies, sendo as mais importantes Piptadenia gonoacantha, Senegalia polyphylla, Machaerium stipitatum, Actinostemon verticillatus e Trichilia casaretti. Por sua vez, a PCA permitiu comprovar a importância das variáveis geográficas, e incorporadas no modelo desenvolvido. As variáveis selecionadas para compor o modelo de regressão linear múltipla foram: número de indivíduos, área basal, longitude e latitude, o qual proporcionou estimativas mais precisas. Esses mesmos gradientes devem ser estudados em escalas regionais e globais, buscando melhorar a capacidade preditiva das variáveis.

\section{AGRADECIMENTOS}

O presente trabalho foi realizado com apoio da Coordenação de Aperfeiçoamento de Pessoal de Nível Superior - Brasil (CAPES) - Código de Financiamento 001. A CEMIG pelo financiamento do projeto Modelo Fitogeográfico da Bacia do Rio Grande GT-456.

\section{REFERÊNCIAS}

ALVARES, C.A. et al. Köppen's climate classification map for Brazil. Meteorologische Zeitschrift, v.22, n.6, p.711-728, 2013.

ARCHANJO, K.M.P.D.A. Análise florística e fitossociológica de fragmentos florestais de mata atlântica no sul do estado do Espírito Santo. 2008. 136p. (Dissertação de mestrado).

BARBOSA, M.R.; THOMAS, W. Biodiversidade, conservação e uso sustentável da Mata Atlântica no Nordeste. Biodiversidade, conservação e uso sustentável da flora do Brasil. Recife: Editora da UFRPE, 2002.

CARVALHO, L.M.T.; SCOLFORO, J.R.S. Inventário Florestal de Minas Gerais: monitoramento da flora nativa 2005-2007. Lavras: Editora UFLA, 2008.

DELGADO, R.C. et al. Seasonality of gross primary production in the Atlantic Forest of Brazil. Global Ecology and Conservation, v.14, p.1-12, 2018 .

FELFILI, J.M. et al. Análise multivariada em estudos de vegetação: Comunicações Técnicas Florestais. Brasília: Universidade Federal de Brasília, 2007.

GIÁCOMO, R.G. et al. Florística e fitossociologia em áreas de campo sujo e cerrado sensu stricto na estação ecológica de Pirapitinga - MG. Ciência Florestal, v.29, p.29-43, 2013.

HIGUCHI, P. et al. Influência de variáveis ambientais sobre o padrão estrutural e florístico do componente arbóreo, em um fragmento de floresta ombrófila mista Montana em Lages, SC. Ciência Florestal, v.22, n.1, p.79-90, 2012.

KASSAMBARA, A.; MUNDT, F. factoextra: Extract and Visualize the Results of Multivariate Data Analyses, 2017.Disponível em: https://CRAN.R-project.org/package=factoextra

LYONS, K.G. et al. Rare Species and Ecosystem Functioning. Conservation Biology, v.19, n.4, p.1019-1024, 2005.

MARTINS, F.R. 0 método dos quadrantes e a fitossociologia de uma floresta residual do interior do Estado de São Paulo: Parque Estadual de Vassununga. 1979. 239p. (Tese de Doutorado).

MEIRA NETO, J.A.A.; MARTINS, F.R. Estrutura da Mata da Silvicultura, uma floresta Estacional Semidecidual Montana no município de Viçosa - MG. Revista Árvore, v.24, n.4, p.459-471, 2000.

MORAIS, V.A. et al. Spatial and vertical distribution of litter and belowground carbon in a brazilian cerrado vegetation. Cerne, v.23, n.1, p.43-52, 2017.

PARRAS-ALCÁNTARA, L. et al. Soil organic carbon along an altitudinal gradient in the Despeñaperros Natural Park, southern Spain. Solid Earth, v.6, p.125-134, 2015.

POORTER, L. et al. Diversity enhances carbon storage in tropical forests. Global Ecology and Biogeography, v.24, p.1314-1328, 2015.

R CORE TEAM. R: A language and environment for statistical computing. Vienna: R Foundation for Statistical Computing, 2018.

RATUCHNE, L.C. et al. Quantificação de carbono florestal em povoamentos de Araucaria angustifolia no sudoeste do estado do Paraná. Ambiência, v.11, n.2, p.321-335, 2015.

REFLORA - Herbário Virtual. Informações. 2019. Disponível em: http://reflora.jbrj.gov.br/reflora/herbarioVirtual/

ROMITELLI, I. Variabilidade espacial nos estoques de carbono em paisagens fragmentadas da Mata Atlântica. 2014. 55p. (Dissertação de mestrado).

SANTOS, R.C. et al. Estoques de volume, biomassa e carbono na madeira de espécies da. Pesquisa Florestal Brasileira, v.36, n.85, p.1-7, 2016.

SCOLFORO, J.R.S. Inventário Florestal de Minas Gerais: equações de volume, peso de matéria seca e carbono para diferentes fitosionomias da flora nativa. Lavras: Editora UFLA, 2008. 
SILVA, A.F.; LEITÃO FILHO, H.F. Composição florística e estrutura de um trecho de mata atlântica de encosta no município de Ubatuba (São Paulo, Brasil). Revista Brasileira de Botânica, v.5, p.43-52, 1982.

SILVA, L.C. et al. Estoques de biomassa e carbono em unidade de conservação no bioma Mata Atlântica. BIOFIX Scientific Journal, v.3, n.2, p 243-251, 2018.

TERRA, M.C.N.S. et al. Influência topo-edafo-climática na vegetação de um fragmento de Mata Atlântica na Serra da Mantiqueira, MG. Revista Ambiente \& Água, v.10, n.4, p.928942, 2015.

TSOZUÉ, D. et al. Catena Changes in soil properties and soil organic carbon stocks along an elevation gradient at Mount Bambouto, Central Africa. Catena, v.175, p.251-262, 2019.

VIANA, R.H.O. et al. Fitossociologia do estrato arbóreo de um fragmento de floresta Estacional Semidecidual Montana - "Mata da Agronomia", Viçosa-MG. Revista de Ciências Ambientais, v.10, n.2, p.141-155, 2016.

VICHARNAKORN, P. et al. Carbon stock assessment using remote sensing and forest inventory data in Savannakhet, Lao PDR. Remote Sensing, v.6, n.6, p.5452-5479, 2014.

WANG, Y. et al. Local-scale spatial variability of soil organic carbon and its stock in the hilly area of the Loess Plateau, China. Quaternary Research, v.73, n.1, p.70-76, 2010. 